\title{
UCRL-CONF-223313
}

LAW RENCE LIVERMORE N A TIO N A L LABORATORY

\section{The Optical Lightpipe as a High-Bandwidth Fusion Diagnostic}

M. J. Moran, R. A. Lerche, G. Mant, V. Yu. Glebov, T. C. Sangster, J. M. Mack

\section{August 2, 2006}

High-Temperature Plasma Diagnostics Conference Williamsburg, VT, United States May 6, 2006 through May 12, 2006 
This document was prepared as an account of work sponsored by an agency of the United States Government. Neither the United States Government nor the University of California nor any of their employees, makes any warranty, express or implied, or assumes any legal liability or responsibility for the accuracy, completeness, or usefulness of any information, apparatus, product, or process disclosed, or represents that its use would not infringe privately owned rights. Reference herein to any specific commercial product, process, or service by trade name, trademark, manufacturer, or otherwise, does not necessarily constitute or imply its endorsement, recommendation, or favoring by the United States Government or the University of California. The views and opinions of authors expressed herein do not necessarily state or reflect those of the United States Government or the University of California, and shall not be used for advertising or product endorsement purposes. 


\author{
The Optical Lightpipe as a \\ High-Bandwidth Fusion Diagnostic \\ M. J. Moran, R. A. Lerche, G. Mant, \\ Lawrence Livermore National Laboratory \\ Livermore, CA 94550 \\ V. Yu. Glebov, T. C. Sangster, \\ Laboratory for Laser Energetics \\ University of Rochester, New York 16423

\section{J. M. Mack, \\ Los Alamos National Laboratory \\ Los Alamos, NM 87545}

\begin{abstract}
A recent series of experiments at the University of Rochester Laboratory for Laser Energetics OMEGA facility studied the feasibility of using radiation-to-light converters and high bandwidth optical signal transmission to remote recording devices as an alternate nuclear diagnostic method. A prototype system included a radiation-to-light converter, a multiple-section light pipe consisting of stainless steel tubes with polished interiors and turning mirrors, and a streak camera or photomultiplier/digitizer combination for signal recording. Several different radiation-to-light converters (scintillators, glasses, plastics, and pressurized $\mathrm{CO}_{2}$ ) performed well and produced predictable optical emissions. The lightpipe transmitted high-bandwidth optical signals to the recording stations. Data were recorded with the streak camera, the photomultiplier/digitizer, and with both recorders simultaneously.
\end{abstract}




\section{Introduction}

The continuing progress of laser-driven inertial confinement fusion (ICF) experiments to higher levels of fusion performance has created a need for a new family of fusion diagnostics. The new diagnostics must function over a wide range of neutron yields (from below $1 \times 10^{16}$ to above $1 \times 10^{19}$ ), be capable of producing signals with sub-nanosecond time resolution, and be sensitive to neutrons and/or energetic photons produced by a burning ICF target. One method that might be able to satisfy these requirements is based on the transmission of optical signals through lightpipes consisting of hollow stainless steel tubes with highly polished interiors. Such a system would consist of a radiation-to-light converter (i.e., a sensor) near the target, an articulated lightpipe with turning mirrors, and a detector/recording device at a remote location that can be shielded from nuclear and electromagnetic radiations. The sensors can be designed for neutrons, $\gamma$ rays, or $\mathrm{x}$ rays, so that a variety of high-bandwidth diagnostic signals can be recorded.

\section{Theory}

The bandwidth of optical signals transmitted through a lightpipe is limited by angular dispersion of light in the tube. The temporal spread of light traveling through a hollow reflective cylinder depends on the angle between the optical wave vectors and the axis of the cylinder. The time $\tau_{\alpha}$ required for a photon with wavenumber $\boldsymbol{k}$ and axial angle $\alpha$ to propagate through a lightpipe of length $\mathrm{L}$ is given by: $\tau_{\alpha}=(\mathrm{L} / \mathrm{c}) / \cos \alpha$, where the optical medium inside the tube is assumed to have a refractive index $n=1$. If the interior of the tube is perfectly smooth, then the angle $\alpha$ will not change, because the normal vector to the cylinder interior has no axial component, and reflections, therefore, will not change the axial component of $\boldsymbol{k}$. 
A beam of photons with an intensity distribution $n(\alpha)$ will have a corresponding range of propagation times, $\tau(\alpha)$, through the lightpipe. If the initial angular distribution of light is isotropic, then an axial solid-angle segment $\Delta \Omega \approx \pi \alpha_{0}{ }^{2}$ (where the maximum angle with the axis is $\alpha_{0}$, and $\alpha_{0}<<1$ ) will propagate with an average temporal spread $\Delta \tau \approx \alpha_{0}{ }^{2} \mathrm{~L} / 4 \mathrm{c}$. For a ten-meter total length and maximum angle of 3 degrees, this formula predicts $\Delta \tau \approx 23$ picoseconds.

The efficiency of light transmission through a lightpipe will be limited by multiple reflections from the interior of the tube. For "s"-polarized light (perpendicular to the plane of incidence) at $3^{\circ}$ degrees from the tube axis the reflectivity is greater than $95 \%$ and increases with decreasing angles to $100 \%$ at $0^{\circ}$ (in the axial direction). However, for "p" polarization, the reflectivity varies linearly with angle from about $70 \%$ at $3^{\circ}$ to $100 \%$ at $0^{\circ} .1,2$

The net transmission of light through the lightpipe will depend on the reflectivities $R_{S}{ }^{N}$ and $R_{p}{ }^{N}$, where $R_{S}\left(R_{p}\right)$ is the $s-(p-)$ polarized reflectivity. $N$, the number of reflections suffered while passing through the lightpipe, is given by: $\mathrm{N}_{\alpha} \approx(\mathrm{L} \sin \alpha) / \mathrm{D}$, where $\mathrm{D}$ is the interior diameter of the tube. The net transmission will be a sum of the contributions from $R_{S}{ }^{N \alpha}$ and $R p^{N \alpha}$ over the angular distribution $\mathrm{n}(\alpha)$ for the respective polarization components. The overall effect is that the net transmission can be good (i.e., greater than 50\%) for light near the lightpipe axis because of the good reflectivities and small numbers of reflections. 
The reflection losses are not necessarily undesirable, because the decreasing reflectivity with increasing off-axis angle has the effect attenuating the off-axis components that increase the temporal dispersion through the light pipe. Thus, the reflection losses have the dual effect of reducing the light transmission through a light pipe, and helping to reduce temporal dispersion at the same time.

Several different mechanisms can be used to convert incident radiation into an optical signal in the light pipe. The simplest method uses a fast scintillator to convert $\mathrm{x}$ rays, $\gamma$ rays or neutrons into an optical signal The emission is relatively efficient: recoil protons from 14-MeV neutrons $\left(\approx 2 \%\right.$ scatter probability for $6-\mathrm{mm}$ thick $\mathrm{BC}-422$ scintillator) each radiate $\approx 3 \times 10^{4}$ photons into $4 \pi$ steradians. $^{3}$ The net effect is about 0.4 photons/neutron radiated into a $3^{\circ}$ half-angle cone. . Since the emission is isotropic, a high-bandwidth system will require apertures and/or lenses and internal reflections to select a nearly on-axis portion of the light.

The so-called "neutron Cherenkov" emission is a multi-step process which relies on inelastic neutron scattering in a transparent medium. ${ }^{4}$ Here, neutrons generate $\gamma$ rays which, in turn, Compton scatter relativistic electrons which then generate optical Cherenkov light as they travel through the medium. The inelastic neutron scattering and Compton events are relatively inefficient (probabilities $\mathrm{P}_{\mathrm{n}, \gamma} \approx 0.03, \mathrm{P}_{\gamma, \mathrm{e}} \approx 0.02$ in $3 \mathrm{~mm}$ of Schott SF6 leaded glass), but the Cherenkov effect can generate large numbers of photons $(\approx 1000$ photons/electron $) .{ }^{5}$ The optical emission is nearly instantaneous, since these processes all occur on a very short time scales, and there is no decay component. The net photon production by this mechanism is about 2000 times 
less efficient than plastic scintillators $\left(\approx 3 \times 10^{-4}\right.$ photons/neutron into a $3^{\circ}$ cone half angle $)$, but it can generate very fast signals.

High-bandwidth optical signals also can be produced by the $16.7-\mathrm{MeV} \gamma$ rays that are generated by DT fusion (with a branching ratio $\approx 10^{-4}$ ). In this case, the $\gamma$ rays Compton scatter relativistic electrons $\mathrm{P}_{\gamma, \mathrm{e}} \approx 0.01$ ) which then pass through a pressurized $\mathrm{CO}_{2}$ cell, where the pressure has been set $(\approx 100$ p.s.i. $\mathrm{x} 100 \mathrm{~cm}$ length) to establish Cherenkov threshold energy of $6 \mathrm{MeV}$, producing broadband optical radiation $(\approx 3$ photons $/ \mathrm{cm})$ in a narrow forward-directed $(\approx 3$ degree cone half angle) beam. ${ }^{6,7}$ The Cherenkov radiation is instantaneous, has an intensity that increases with decreasing wavelength, has no decay component, and has an integrated efficiency of about 1.5 photons $/ \gamma$ ray (equivalent to $\approx 1.5 \times 10^{-4}$ photons per DT neutron).

\section{Experiment}

A series of experiments at the Laboratory for Laser Energetics OMEGA facility tested the lightpipe diagnostic concept. BC-422 scintillator, fused silica or Lucite radiators (for neutron Cherenkov radiation), and $\mathrm{CO}_{2}$ threshold Cherenkov sensors were used to record neutron and $\gamma$ ray signals from a series of DT implosions with yields from 0.5 to $5.0 \times 10^{13}$ DT neutrons. Figure 1 is a sketch of one experimental arrangement. The lightpipe sensor was inserted into a diagnostic well on the OMEGA target chamber to a position $22 \mathrm{~cm}$ from the target chamber center (TCC), without penetrating into the chamber vacuum. Light from the sensor propagated along a 10-meter path that was defined by a series of lightpipes (1-inch i.d. stainless steel tubing) and two turning mirrors. The light was detected by a Photek microchannel-plate photomultiplier (MCP), a streak camera, or an $\mathrm{MCP} /$ streak camera combination. Light was focused onto the 
photocathodes with 50-mm focal length fused-silica lenses. Electrical signals from the MCP were recorded by transient digitizers.

\section{Results}

Figure 2 shows the cropped neutron scintillator signal and timing fiducial streak camera records and signal lineouts for a DT capsule implosion having a yield of $2.9 \times 10^{13}$ neutrons. The signal was generated by unquenched Eljen 232 plastic scintillator, and the streak camera was set for an approximately 10-ns streak duration. The signal-to-noise ratio in the data was very good, and few "stars" from neutron background were evident in the data. This example was recorded with an ND-1 neutral density filter in front of the streak camera. These data are similar to the results that have been achieved previously with the neutron temporal diagnostic (NTD) that uses a scintillator which is coupled to a streak camera in the immediate vicinity of the target chamber. The advantage of the present system is that it records high bandwidth signals from scintillators close to TCC on a streak camera at a remote location that can be shielded from intense neutron backgrounds.

Figure 3 shows the cropped neutron Cherenkov signal and timing fiducial streak camera records and signal lineouts for a DT capsule implosion having a yield of $7.1 \times 10^{13}$ neutrons. The signal was generated by a 3-mm of Schott SF6 leaded glass, and the streak camera again was set for an approximately 10-ns streak duration. Several "stars" from neutron background are evident in the data. The 370-psec FWHM of the streak signal lineout is dominated by the resolution limit of the streak camera for this streak speed.

The vertical heights of the streaks is about $1.2 \mathrm{~mm}$ in both in Figs. 2 and 3. This direction is parallel to the slit on the front of the streak camera, so it is an indication of the spot size of the 
focused light. The 1.2-mm height, produced by the $50-\mathrm{mm}$ lens, indicates that the angular spread of the light incident on the lens had a half-width of about 0.7 degrees. Since the neutron Cherenkov light is isotropic, this suggests that off-axis light was severely attenuated by multiple reflections in the lightpipe. However, the 0.7 -degree half angle corresponds to an average temporal dispersion of about $1.5 \mathrm{psec}$ through the 10-m lightpipe, although the data above do not have the bandwidth to demonstrate this ultimate time response.

Figure 4 shows a transient digitizer recording of the signal from the $\mathrm{CO}_{2}$ threshold Cherenkov cell for a DT implosion with yield $=7.1 \times 10^{13}$. This signal was recorded by an MCP (with gain $=1000$ ) which received a $10 \%$ pickoff of the light that was focused on the streak camera. The signal at $208 \mathrm{~ns}$ is the fast Cherenkov signal from the $16.7 \mathrm{MeV} \gamma$ rays produced by DT fusion. The signal at $211 \mathrm{~ns}$ is associated with the arrival of neutrons at the $\mathrm{CO}_{2}$ cell, but the specific detection mechanism has not been identified. The identification of the 208-ns signal with the $16.7 \mathrm{MeV} \gamma$ rays was made by comparison of absolute timing with neutron induced signals, and by correlation of signal amplitude with fusion yield.

Figure 5 displays the distribution of relative times for a high-bandwidth neutron detector (CVD diamond detector) and the fusion $\gamma$-ray signal from the lightpipe threshold Cherenkov sensor. The data demonstrate that the timing of the $\gamma$-ray signal is accurately correlated with the timing of the neutron pulse. The standard deviation of $16 \mathrm{psec}$ becomes $10 \mathrm{psec}$ if shot \#9 is excluded from the data (The Shot \#9 signal had an extra background component that affected the time assigned to the signal).

V. Discussion 
The data above demonstrate that an optical lightpipe can serve as the basis of high-bandwidth diagnostics in high-background ICF environments. These initial experiments used a very simple design that was intended to test the basic usefulness of this signal transmission method. There are features that could be added to the design, such as nickel plating of the interior of the lightpipe, or using lenses to collimate light in the lightpipe, that have the potential to improve the performance of the system. In any case, the present demonstration was successful: The sensors can be designed to respond selectively to different portions of the radiation spectrum, the signals have predictable amplitudes and high bandwidths, and they can be recorded at remote locations that can be shielded from background radiation. and high bandwidths, and they can be recorded at remote locations that can be shielded from background radiation.

This work was performed under the auspices of the U.S. Department of Energy by the University of California, Lawrence Livermore National Laboratory under contract No. W-7405-Eng-48. 
VI. References

1) Karlsson, B. and Ribbing, C.G., J. Appl Physics 53(9), (1982) p. 5349.

2) Brouwer, W. and McClure, N.R., American Institute of Physics Handbook, Third Edition, Section 6g, p. 6-122, McGraw-Hill (1972).

3) Knoll, G.F., Radiation Detection and Measurement, Third Edition, (John Wiley and Sons, New York, p. 225 (2000).

4) Clark, D.L., Lawrence Livermore National Laboratory report UCID-19397, 1982.

5) Jelley, J.V., Cherenkov Radiation and its Applications, (Permagon, New York, 1958).

6) M.J. Moran, Rev. Sci. Inst., 56, (1985) 1066.

7) J. Mack, R.R. Berggrena, S.E. Caldwella, S.C. Evansa, J.R. Faulkner Jr., R.A. Lercheb, J.A. Oertela, C.S. Young" Nuc. Inst. and Methods in Physics Research A 513 (2003) 566-572. 


\section{FIGURE CAPTIONS}

Figure 1 Sketch of experimental arrangement The lightpipe allows signals to be recorded electronically in a well-shielded location about ten meters from target chamber center.

Figure 2 Streak data for scintillator/neutron pulse

The 3-ns optical streak image (center) shows the fidu timing pulse train at the top and the scintillator pulse below. Lineouts on a nanosecond time scale for the fidu and scintillator are shown on the top and bottom, respectively.

Figure 3 Streak data for neutron Cherenkov/ neutron pulse The 10-ns window optical streak of the fidu and neutron signals are shown with the same layout as in Fig. 2. Here, the 368 psec FWHM represents a convolution of the fusion burn width and the streak camera resolution.

Figure 4 Digitizer recording of threshold Cherenkov $\gamma$-ray signal

The digitizer recording shows that the distinct Cherenkov signal at $208.5 \mathrm{~ns}$ (solid) precedes the neutron Cherenkov signal by about 2 ns. With the Cox removed from the detector cell, only the neutron Cherenkov signal (dashed) remains.

Figure 5 Relative timing of neutron and threshold Cherenkov signals Identification of the $\mathrm{CO}_{2}$ threshold Cherenkov g-ray signal is confirmed by temporal correlation with the neutron signal (shown above) and by amplitude correlation with neutron yield. 


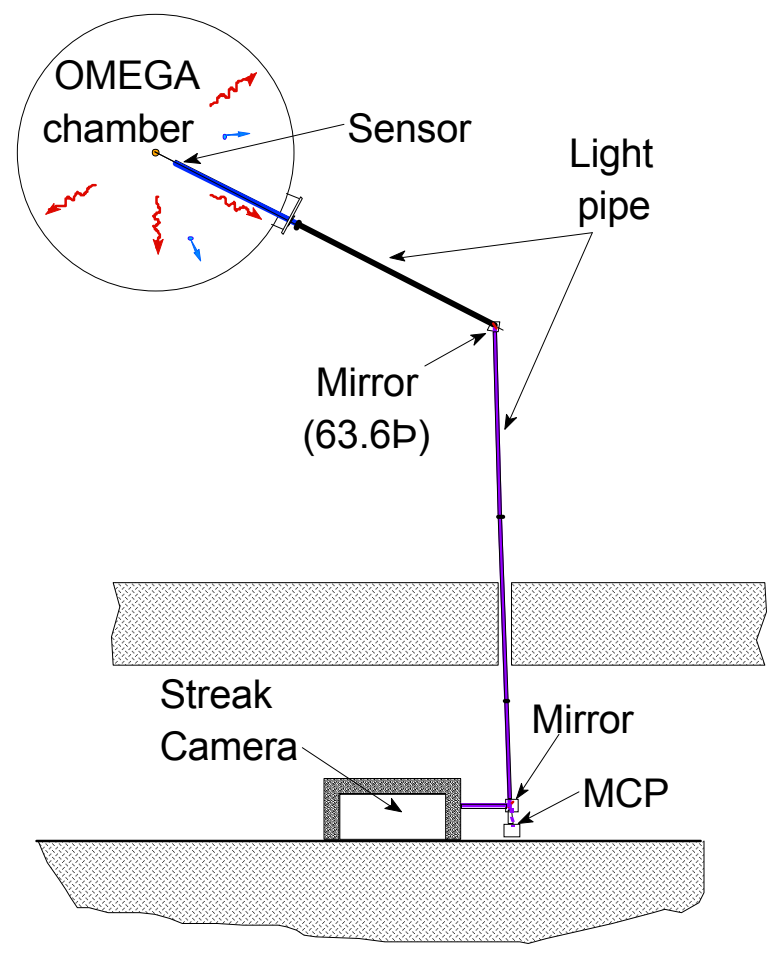

Figure 1 


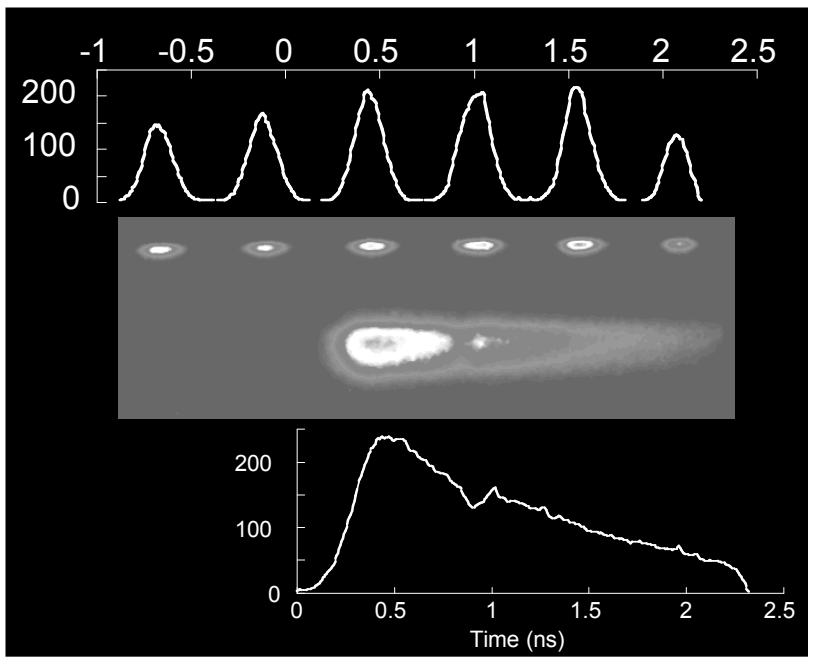

Figure 2 


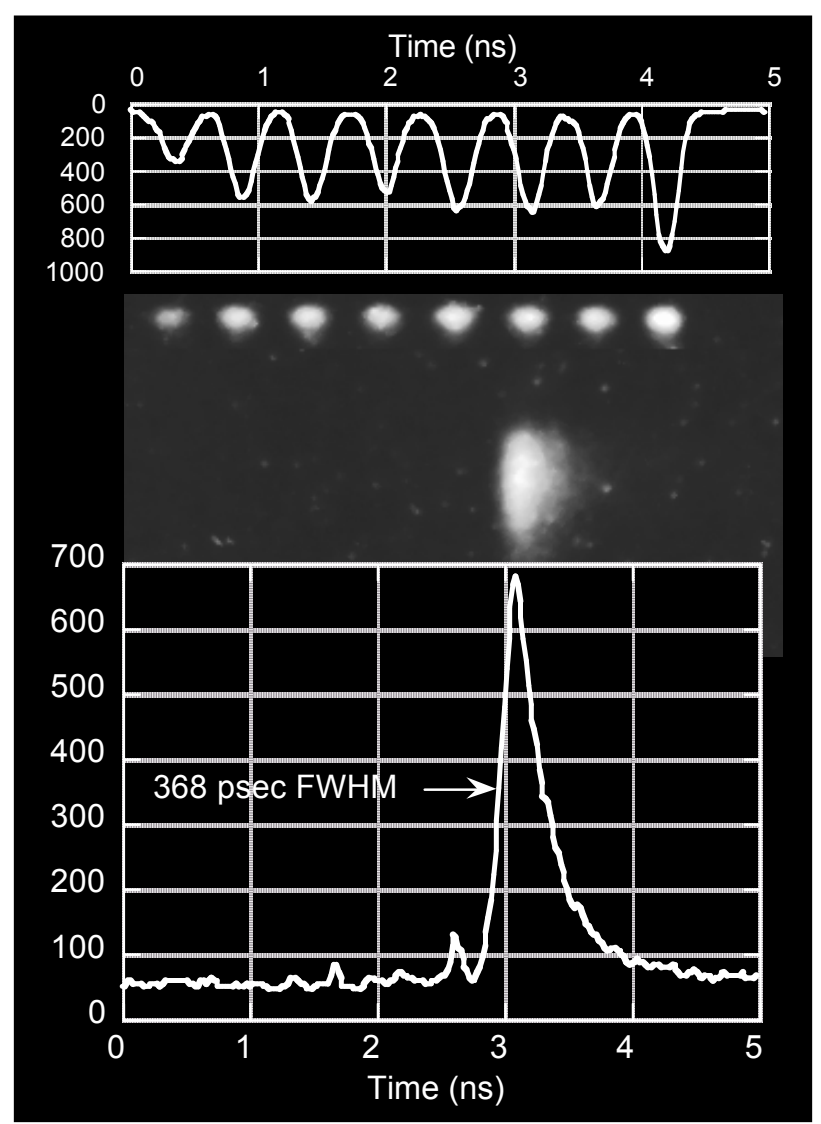

Figure 3 


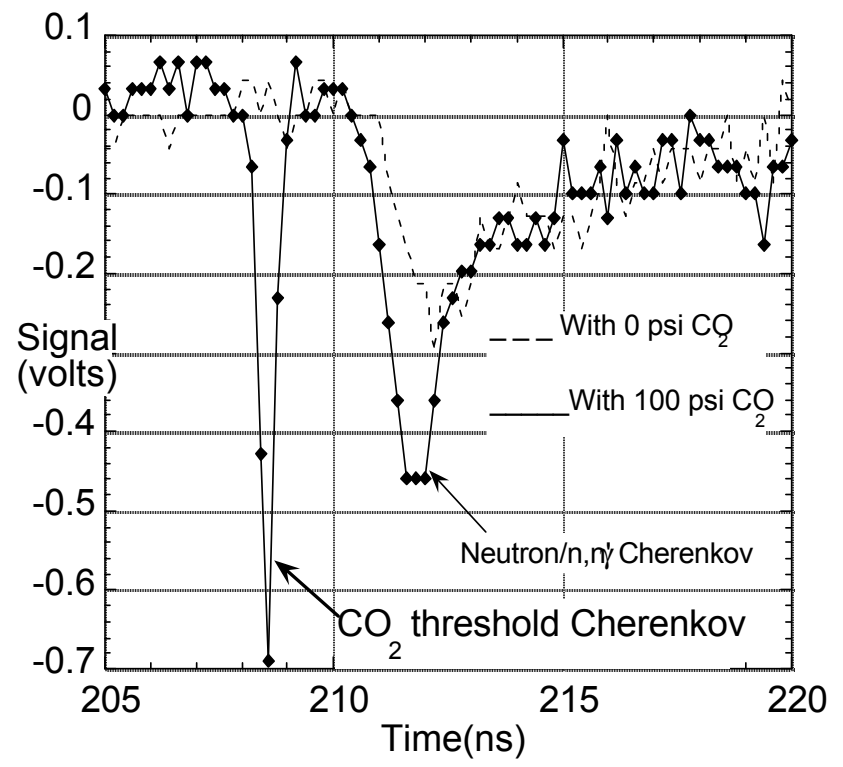

Figure 4 


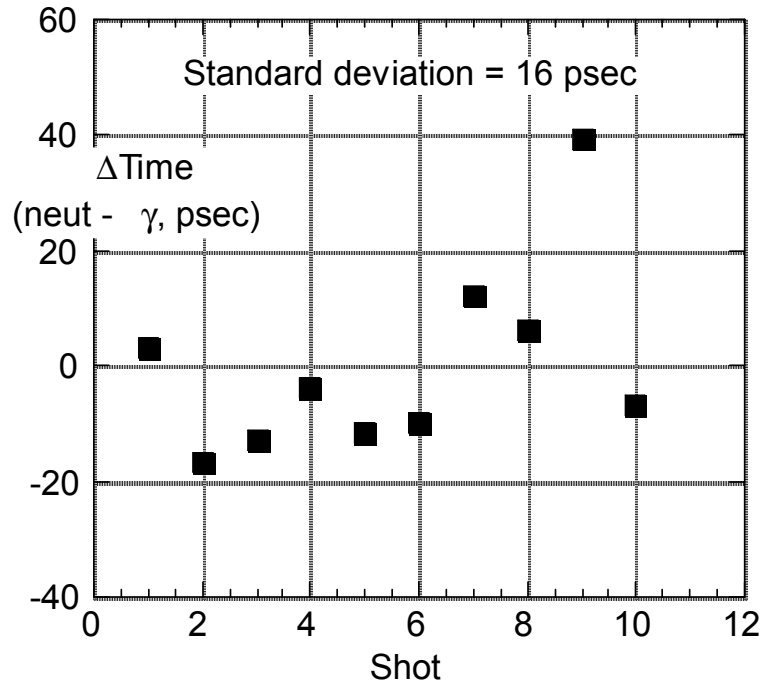

Figure 5 